\title{
Comparative Divertor-Transport Study for Helical Devices
}

\author{
Y. Feng 1), M. Kobayashi 2), F. Sardei 1), S. Masuzaki 2), J. Kisslinger 1), T. \\ Morisaki 2), P. Grigull 1), H. Yamada 2), K. McCormick 1), N. Ohyabu 2), R. \\ König 1), I. Yamada 2), L. Giannone 1), K. Narihara 2), U. Wenzel 1), S. Morita \\ 2), H. Thomsen 1), J. Miyazawa 2), D. Hildebrandt 1), T. Watanabe 2), F. \\ Wagner 1), N. Ashikawa 2), K. Ida 2), A. Komori 2), O. Motojima 2), Y. \\ Nakamura 2), B.J. Peterson 2), K. Sato 2), M. Shoji 1), N. Tamura 2), M. \\ Tokitani 2), the LHD experimental group 2)
}

1) Max-Planck-Institut für Plasmaphysik, Euratom Association, Germany

2) National Institute for Fusion Science, Toki, Japan

\begin{abstract}
Using the island divertors (IDs) of W7-AS and W7-X and the helical divertor (HD) of LHD as examples, the paper presents a comparative divertor transport study for three typical helical devices of different machine-size following two distinct divertor concepts, aiming at identifying common physics issues/effects for mutual validation and combined studies. Based on EMC3/EIRENE simulations supported by experimental results, the paper first reviews and compares the essential transport features of the W7-AS ID and the LHD HD in order to build a base and framework for a predictive study of W7-X. The fundamental role of low-order magnetic islands in both divertor concepts is emphasised. Preliminary EMC3/EIRENE simulation results for W7-X are presented and discussed with respect to W7-AS and LHD in order to show how the individual field and divertor topologies affect the divertor transport and performance. For instance, a high recycling regime, which is absent from W7-AS and LHD, is predicted to exist for W7-X. The paper focuses on identifying and understanding the role of divertors for high density plasma operations in helical devices. In this regard, special attention is paid to investigating the divertor function for controlling intrinsic impurities. Impurity transport behaviour and wall-sputtering processes of CX-neutrals are studied under different divertor plasma conditions. A divertor retention effect on intrinsic impurities at high SOL collisonalities is predicted for all the three devices. The required SOL plasma conditions and the underlying mechanisms are analysed in detail. Numerical results are discussed in conjunction with the experimental observations for high density divertor plasmas in W7-AS and LHD. Different SOL transport regimes are numerically identified for the standard divertor configuration of W7-X and the possible consequences on high density plasmas are assessed. All the EMC3-EIRENE simulations presented in this paper are based on vacuum fields and comparisons with local diagnostics are made for low- $ß$ plasmas.
\end{abstract}

PACS numbers: $\mathrm{xxxxxx}$

\section{Introduction}

Unlike the standard poloidal-field divertor in tokamaks, divertor concepts presently investigated in stellarators are based on specific edge magnetic field structures intrinsically available in each device [1]. Typical examples are the island divertor for the advanced lowshear stellarators W7-AS [2] and W7-X [3], and the helical divertor for the high-shear, largest heliotron-type device LHD [4]. The large differences in machine type and size, in combination with the inherent high configurational flexibility of each helical device, open a broad configuration spectrum for exploring divertor solutions for plasma exhaust in helical devices, introducing however, besides the inherent three-dimensionality, another additional 
independent parameter, i.e. the divertor configuration, into the divertor physics within the stellarator community. Inter-machine comparisons are important not only for revealing the configurational dependence of divertor functionality and performance but also for identifying common interesting physics effects for mutual validation and combined studies. For this, the EMC3/EIRENE code [5, 6] provides a useful interpretative and predictive tool. On the other hand, an enriched divertor database contributed from different machines provides a broader basis for code validation to improve the code prediction capability for next-step devices.

Comparison between the W7-AS island divertor (ID) and the LHD helical divertor (HD) has been made for several specific topics. On an experimental basis, the essential detachment behaviour in LHD has been compared with that in W7-AS, showing that sustained detachment can be achieved in both devices, with the detached plasmas, however, evolving through different paths to different states [7]. Using the W7-AS ID as reference, the momentum transport in the HD of LHD has been studied using the EMC3/EIRENE code in comparison with experimental results [8], concluding that the suppression of a high recycling regime in LHD is due to a geometric momentum loss process similar to that claimed for W7AS [9]. This finding has triggered detailed studies to find the common geometric elements governing the SOL transport in both devices, having identified the fundamental role of the low-order islands in both divertor concepts [10]. From this starting point, the island screening potential of intrinsic impurities, which is shown by EMC3/EIRENE simulations for the ID of W7-AS at high SOL collisionalities [11], was also examined and has been found for the HD of LHD [12] as well. The numerical results are consistent with the experimental observations of the drop of Fe-line radiation in high density divertor plasmas in both devices [13, 14]. Direct evidence has been recently provided by the edge spectroscopic measurements on LHD, which show, with increasing plasma density, a clear relative shift of carbon line emission from high to low charge states populated at different SOL depths [15].

The first-step results from the joint-studies between W7-AS and LHD are promising, encouraging a predictive study for W7-X. The paper presents the first EMC3/EIRENE simulation results for the standard ID of $\mathrm{W} 7-\mathrm{X}$ and compares the basic divertor transport features of three typical helical devices of different size and geometry.

It is to be pointed out that all the EMC3-EIRENE simulations presented in this paper are based on vacuum field configurations. There are different reasons for this. First, the paper focuses on general transport effects and physics related to the basic SOL topologies in each device and not restricted to specific discharges. Changes in leading geometric parameters, such as the island width and the divertor-relevant field-line pitch, induced by finite $\beta$-effects and the related plasma currents can well be realized and represented by vacuum configurations. Second, there are no systematic experimental studies of equilibrium divertor configurations having been made for helical devices. As an essential input for EMC3EIRENE, any uncertainties in divertor configuration will enter the transport processes, making the interpretation of the mixed results very difficult. Third, direct comparisons with local diagnostics would be made for low- $\beta$ plasmas until experimentally-verified equilibrium divertor configurations are available.

Section 2 illustrates the principles and geometries of the W7-AS and W7-X IDs and the LHD HD, with the leading geometric parameters determining the SOL transport being listed for comparison. The fundamental role of the low-order magnetic islands in both divertor concepts is exhibited in section 3, and the essential plasma transport behaviour associated with magnetic islands is presented in section 4. Section 5 addresses the divertor function for controlling intrinsic impurities - a key element for obtaining high density plasmas in helical devices. The impact of the SOL plasma on the high-energetic wall-sputtering CX-neutrals is shown in W7-AS and LHD. Particularly, the impurity transport behaviour under different SOL plasma conditions is investigated and an impurity retention effect is presented. The 
underlying physics is explained. Section 6 gives an analysis of favourable SOL plasma conditions for achieving high density plasmas in helical devices by self-consistently taking impurity transport and radiation, recycling and particle fuelling into account. A summary incorporated in a general discussion is given in section 7 .

\section{Divertor concept and geometry}

W7-AS and W7-X are low-shear stellarators having five-fold field symmetry. The low-shear in the Wendelstein-7 series allows large island formation as the radial size of the islands scales as $r_{i} \propto \sqrt{R b_{m n} / m \boldsymbol{z}^{\prime}}$ where $\mathrm{R}$ is major radius, $b_{m n}$ the resonant radial perturbation field normalized to the toroidal field, $m$ the poloidal mode number and $l^{\prime}$ the shear. Within the respective $l$-ranges available in each device, a number of low-order island chains of different poloidal mode numbers are suitable for the ID. The so-called standard ID configurations for W7-AS and W7$\mathrm{X}$ are based on the $5 / 9$ and $5 / 5$
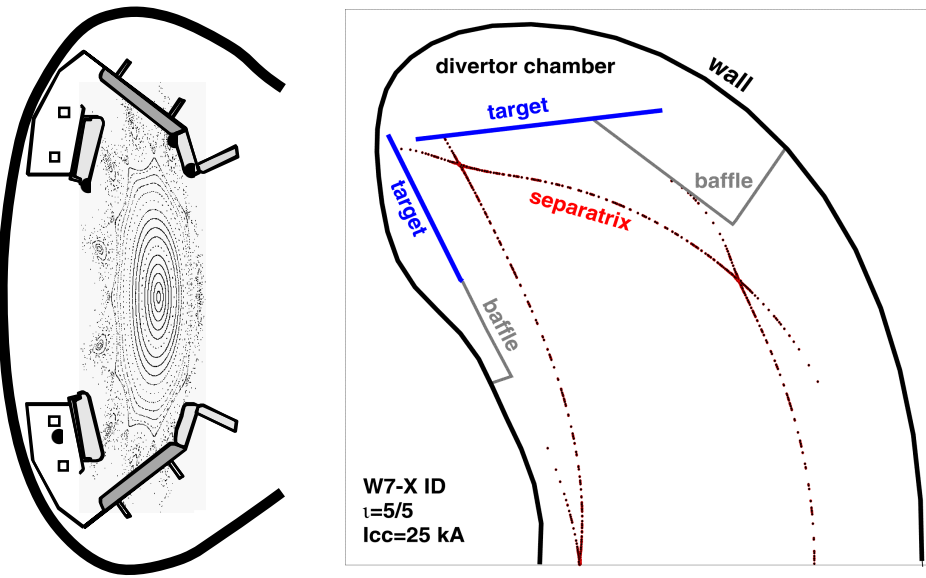

Fig.1 The standard island divertor configurations for W7-AS (left) and W7-X (right, only a half) with respective nine and five islands at the edge. The two devices follow the same ID concept, equipped with similar divertor modules shaped and positioned according to the machine symmetry.

island chains, respectively, as shown in Figure 1.That particles and energy entering the islands across the inner separatrix are guided by the open field lines inside the islands towards the targets positioned at the outer separatrix over an appropriate distance away from the confinement core is the functional principle of the ID concept. The island size and the internal field-line pitch, which affect the island divertor transport, can be adjusted externally by ten control coils installed on each machine.

LHD is the largest heliotron-type device with 10 field periods and has a large shear, especially at the edge. Two different edge configurations are being explored for divertor application, i.e. the local island divertor (LID) and the HD. The former is based on a 1/1magetic island generated by external perturbation coils, whereas the latter relies on a stochastic layer intrinsically existing at the edge. Here, we choose the HD as an example for our comparative studies. Divertor transport studies and numerical and experimental results for the LID are refereed to in Refs [16-19]. The rotational transform in the edge region of the HD configurations covers many resonances which overlap each other, forming a stochastic layer of $\sim 10 \mathrm{~cm}$ thickness. Unlike the single island chains in W7-AS and W7-X, the stochastic SOL in LHD exhibits a complex field structure characterized by the coexistence of remnant magnetic islands, stochastic fields and edge surface layers. In the outermost region close to the wall, the increased poloidal field components of the two helical coils create 4 divertor legs which are cut by graphite targets positioned just in front of the wall (figure 2), forming

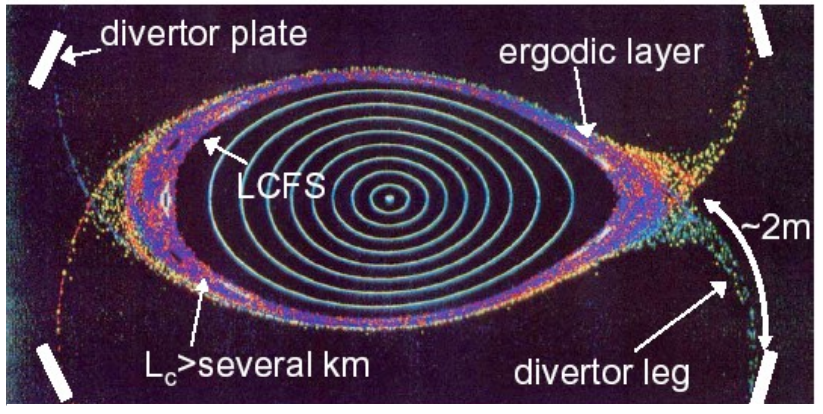

Fig. 2 Field and divertor geometry of the helical divertor in $L H D$. 
a divertor configuration similar to the double-null configuration in tokamaks. However, the resulting short connection lengths between the ' $\mathrm{X}$ '-points and the targets, which are $\sim 2 \mathrm{~m}$ on average, make the divertor legs transparent for recycling neutrals, at least in the present open divertor configuration [8]. In contrast, the pre-X-point stochastic layer is much thicker and, hence, is expected to largely determine the divertor performance of the open HD. In the following, our attention will be paid only to the stochastic layer.

The divertor-relevant geometric parameters for the respective standard ID configurations in W7-AS and W7-X are listed in Table 1, where the last four parameters have

Table 1: Comparison of island-divertor-relevant geometric parameters between W7-AS and W7-X.

$L_{c}=$ target-target connection length, $\Delta x=$ target-core distance, $\Delta y=$ poloidal island width, $\Theta=$ pitch

\begin{tabular}{|l|c|c|c|c|c|c|c|}
\hline & $\mathrm{R}(\mathrm{m})$ & $\mathrm{a}(\mathrm{m})$ & $\mathrm{\imath}$ & $\mathrm{L}_{\mathrm{c}}(\mathrm{m})$ & $\Delta \mathrm{x}(\mathrm{cm})$ & $\Delta \mathrm{y}(\mathrm{cm})$ & $\Theta\left(10^{-3}\right)$ \\
\hline W7-AS & 2.0 & 0.14 & $5 / 9$ & $\sim 100$ & $\sim 4$ & $\sim 10$ & $\sim 1-1.5$ \\
\hline W7-X & 5.5 & 0.50 & $5 / 5$ & $\sim 180$ & $\sim 7-8$ & $\sim 60$ & $\sim 2-3$ \\
\hline
\end{tabular}

only a representative meaning because of the complex 3D SOL structures. The field line pitch $\Theta$, which defines the relative perpendicular displacement of a field line towards the targets per field-line length, is estimated for the divertor regions by measuring the parallel and perpendicular X-point-to-target distances $L_{c, x-t}$ (connection length of the X-point) and $\Delta x$, i.e. $\Theta=\Delta x / L_{c, x-t .}$. LHD has a major radius of $3.9 \mathrm{~m}$ and a minor radius of $0.6 \mathrm{~m}$. The rotational transform in the stochastic layer varies from $\sim 10 / 7$ to $10 / 2$. The open field lines in the stochastic layer exhibit a rather complex evolution structure, yielding a connection length contour ranging from several $\mathrm{m}$ to several $\mathrm{km}$. The outermost region is dominated by field lines of short connection lengths. There exist, however, multiple edge surfaces [4] filled by long field lines of several $100 \mathrm{~m}$ connection length, forming the main plasma parallel transport channels across the stochastic layer. Thus, the characteristic perpendicular-to-parallel transport scalelength ratio in LHD, i.e. $10 \mathrm{~cm}$ SOL thickness divided by several $100 \mathrm{~m}$ connection length, is even smaller than those in the IDs of W7-AS and W7-X.

\section{Role of low-order magnetic islands}

Plasma transport in the island SOL of W7AS can well be explained in terms of regular magnetic islands [9]. W7-X follows the same ID concept, only with much larger magnetic islands. Thus, the meaning of a W7-AS-W7-X comparison is straightforward without the need of justification. In contrast, as shown by figure 2, the LHD HD presents a rather

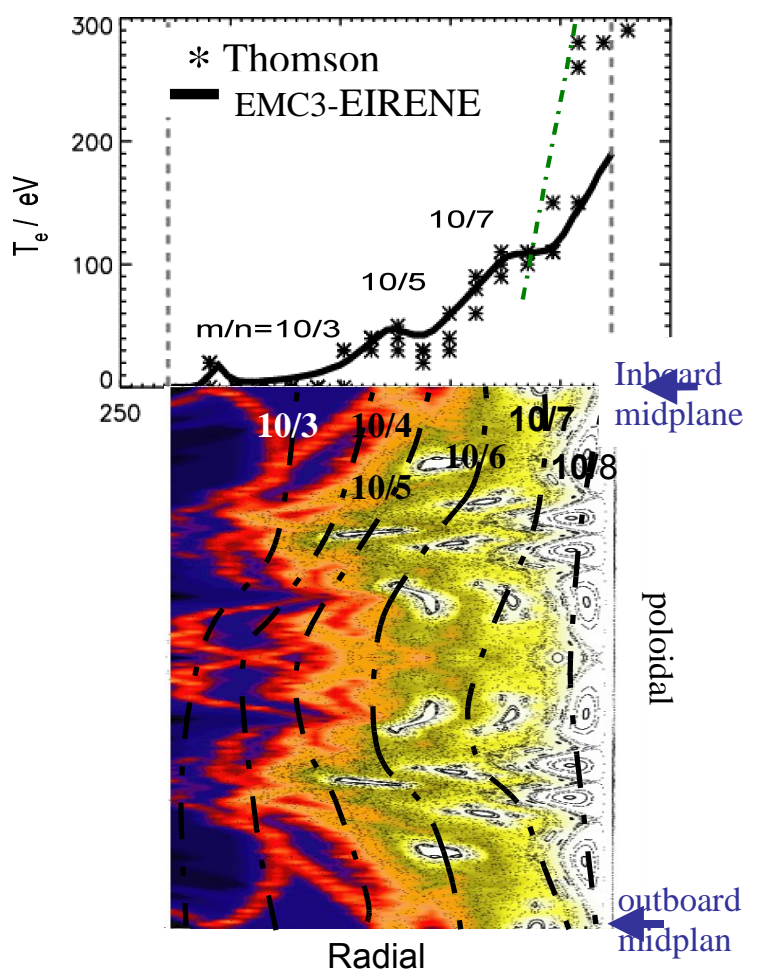

Fig.3. Comparison of Te-profiles in LHD between simulations and Thomson measurements (top) and a radially-zoomed connection length contour with an overlying Poincare plot (bottom) over half poloidal field period at a toroidal location where the long axis of the elliptical cross-section lies horizontally. The dot-dashed lines sketch the locations of the low-order magnetic islands. 
complex field structure. From the geometry point of view, it is not obvious on what basis the HD should be compared with the IDs. For this reason, detailed EMC3/EIRENE simulations have been performed [10] in order first to clarify how and to what extent the plasma as fluid follows the complex field structure in LHD.

The following transport analysis is based on the simulation results given in Ref. [10] for a vacuum configuration with $\mathrm{R}=3.75 \mathrm{~m}$. The discharges selected here for comparison have typical $<\beta>$-values of less than $0.5 \%$ (full field with NBI-heating powers $<8 \mathrm{MW}$ at $\bar{n}$ $<8 \times 10^{19} \mathrm{~m}^{-3}$ ). The lower picture of figure 3 shows the field line connection length contour together with Poincare plots on the toroidal position of a Thomson scattering measurement. The SOL begins with the 10/7 island chain in which the field lines in most of the region have already become irregular, with only small island cores remaining. A similar situation is also presented by the neighbouring 10/6 island chain. Moving outwards, closed island cores vanish and Poincare-plots show a strong irregularity of the field lines, thus indicating that the field is stochastic. On the other hand, the underlying connection length contour shows a strong field-line correlation even in the outer region without remnant island cores. The connection length contour reflects actually the basic field structure of low-order resonances. Indeed, all the low-order modes expected within the given l-range can be identified [10]. The upper picture of figure 3 shows the calculated and the Thomson-scattering $\mathrm{T}_{\mathrm{e}}$-profiles along the inboard midplane. Both the code and the Thomson results clearly show the impact of the low-order 10/7, 10/5 and 10/3 magnetic islands on electron energy transport. The 10/8, 10/6 and 10/4 mode structures have a poloidally-shifted phase distribution, with the X-points being on the midplane. This is the reason why they are not reflected by the $T_{e}$-profiles. On the other hand, the good agreement between the calculated and the Thomson $\mathrm{T}_{\mathrm{e}}$-profiles throughout the stochastic layer justifies the vacuum configuration approximation made in the modeling. In addition, the code shows a strong correlation of parallel plasma flows with the island structure where positive and negative flows surround the O-points [10]. Even for the mode structures without a closed island core, flow channels residing on the island chains are still identifiable. Thus, it is to conclude that the plasma transport in the stochastic layer is governed by the loworder islands, although the relative importance of the stochastic effects cannot yet be quantified.

\section{Transport characteristics}

In the context of the fluid approximation, particle transport along field lines is governed by a classical convective process, with the parallel

flow velocity being determined by momentum balance. Momentum transport in both W7-AS and LHD divertor configurations is characterized by friction between opposite flows in different parts of the magnetic islands, which gives rise to significant momentum loss and thereby breaks pressure conservation along open field lines already under low-density, hightemperature conditions without intensive plasma-neutral interaction. In W7-AS, interaction between opposite flows occurs in both the poloidal and radial directions [9], while in LHD the radial approach of counterflows residing on neighbouring island chains

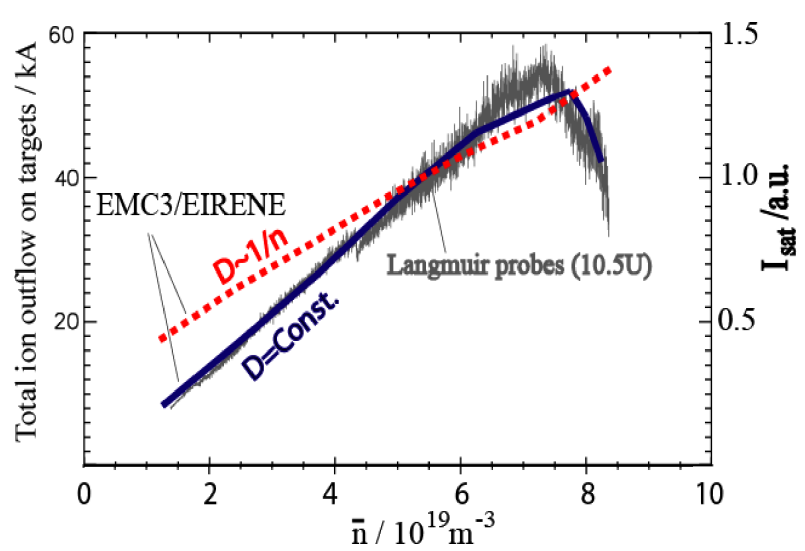

Fig. 4. Ion saturation currents increase linearly with plasma density in LHD. This linear-dependence can well be reproduced by the $3 D$ code, independent on the D-ansatz. 
is the main reason for flow damping $[8,10]$. As a consequence, a high recycling regime as observed in tokamaks does not take place in the island/stochastic SOLs of W7-AS and LHD and is also not expected by modeling. As an example for LHD, figure 4 shows a typical density dependence of the ion saturation currents measured by target Langmuir probes. In agreement with $\mathrm{H}_{\mathrm{a}}$-signals, $\mathrm{I}_{\text {sat }}$ grows linearly with increasing line average density up to a rollover point. This linear behavior can be well reproduced by the EMC3/EIRENE code, insensitively to the selected density dependence of the cross-field diffusion coefficient. Nevertheless, the use of the constant $\mathrm{D}=0.5 \mathrm{~m}^{2} / \mathrm{s}$ results in an ion flow slope matching better that of $\mathrm{I}_{\text {sat }}$ from the probes.

The larger field line pitch in W7-X decreases the perpendicular-to-parallel transport ratio, generally reducing the perpendicular viscous transport, assumed that $\mathrm{D}$ does not change very much with the machine size. Moreover, the large poloidal extension of the W7-X islands avoids cross-field frictional momentum interaction between adjacent island fans. This suggests that the SOL transport in W7$\mathrm{X}$ should behave differently from that in W7-AS and LHD. This is indeed shown by EMC3/EIRENE simulations [20], as demonstrated in figures 5 and 6 , where the W7-

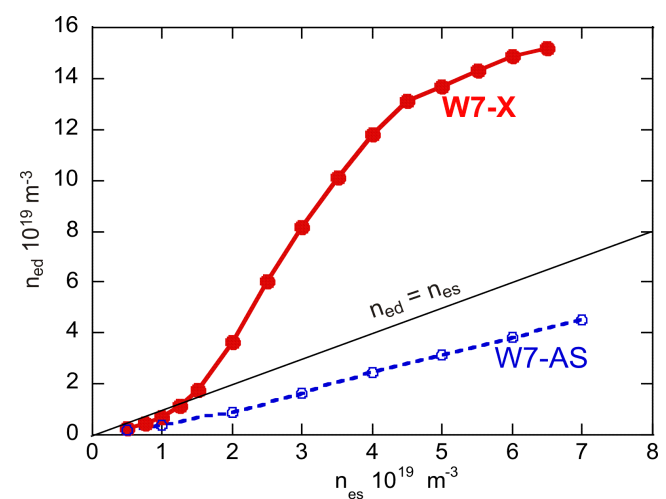

Fig.5: Comparison of $n_{e s}$-dependences of the downstream density $n_{\text {ed }}$ between W7-AS

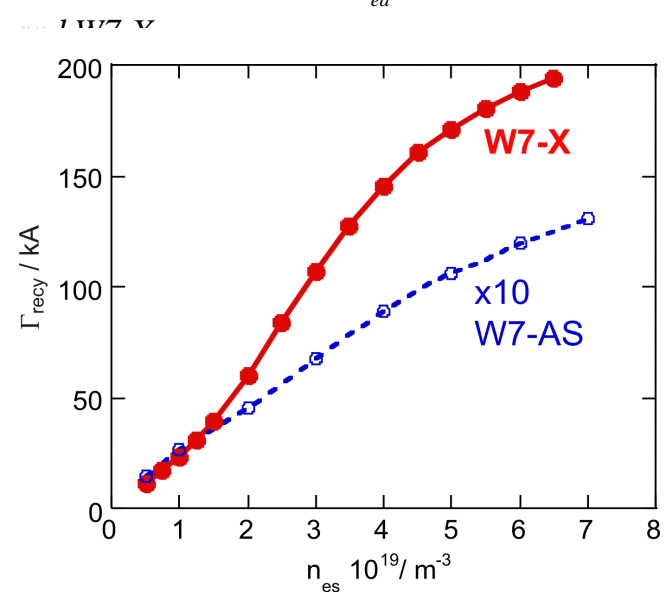

Fig.6: Responses of the total recycling flux $\Gamma_{\text {recy }}$ to the separatrix density $n_{\text {es }}$ predicted for the respective W7-AS and W7-X standard island divertors. AS simulations serve as references. Simulations are carried out for pure hydrogen plasmas without impurities, for the respective standard ID configurations based on vacuum field. The SOL power for W7-AS is taken to be $1 \mathrm{MW}$ which is up-scaled with the area of the LCFS to $10 \mathrm{MW}$ for W7-X. The cross-field transport coefficients are set as $\mathrm{D}=1 \mathrm{~m}^{2} / \mathrm{s}$ and $\chi_{\mathrm{i}}=\chi_{\mathrm{e}}=3 \times \mathrm{D}$ which hold for both devices. At low plasma separatrix density $\mathrm{n}_{\mathrm{es}}$ - the average density on the LCFS, both devices show linear growths of the recycling flux $\Gamma_{\text {recy }}$ and the downstream density $n_{\text {ed }}$ with increasing $n_{\text {es }}$ up to $\sim 1.3 \times 10^{19} \mathrm{~m}^{-3}$. Then, the W7-X results suddenly depart from the linear behaviour further followed by W7AS. In this range, $\mathrm{n}_{\mathrm{ed}}$ in W7-X approaches a scaling of $\mathrm{n}_{\mathrm{ed}} \sim \mathrm{n}_{\mathrm{es}}{ }^{3}$ and greatly exceeds $\mathrm{n}_{\mathrm{es}}$, as it usually behaves in a high recycling regime found in tokamaks. W7-X is the first helical device for which a high recycling regime is predicted not only by the $3 \mathrm{D}$ code but also by previous 2D modelling approximations [21].

\section{Divertor function on controlling intrinsic impurities}

Impurity transport analyses based on TJ-II, W7-AS and LHD experimental results yield a common general tendency, i.e. that impurity confinement improves towards high densities [22]. Thus, controlling the impurity influx into the core plays a crucial role in purifying the confinement region to allow high plasma densities. In fact, reducing the impurity release from plasma facing components and preventing impurities from transporting towards the 
confinement core belong to the main design objectives of a divertor. Related theoretical research made for W7-AS so far was concentrated on exploring favourable divertor plasma conditions for reducing the impurity influx. The work was trigged by the experimental observation of a strong drop of Fe-content in the HDH (high density $\mathrm{H}$ )-mode [23] in W7-AS [13]. The ELM-free HDH-mode does not suffer from a radiation collapse as usually observed in quiescent $\mathrm{H}$-mode in W7-AS. Consistent with the drop in Fe-content, Al-LBO measurements show a strong reduction of $\tau_{\mathrm{Al}}$ in the HDH-regime [24]. As a supplementary factor EMC3-EIRENE modeling shows that the edge magnetic islands under the HDH conditions have a screening effect on intrinsic impurities [11]. Both effects lead to plasma purification. Experimental separation of the two effects was beyond the diagnostic capability available on W7-AS at that time. Hence, the island impurity retention effect remains a theoretical issue for W7-AS to be further investigated at W7-X. Fortunately, the work could be continued in LHD - the only helical device presently operated with a divertor. A similar impurity screening effect has been also predicted for the stochastic layer in LHD at high SOL collisionalities [12]. In fact, LHD experiments show also a reduction of Fe-content when plasma density, for a given heating power, is increased beyond a certain level [14]. Nevertheless, in contrast to the HDH-mode in W7-AS, the Fe-purified high-density plasmas in LHD do not benefit from a reduced impurity confinement in the core for which there is no experimental evidence. Thus, the SOL impurity screening effect predicted by the 3D code remains the only explanation for the experimental observations in LHD. Indeed, the edge spectroscopic measurements on LHD have recently confirmed the SOL impurity retention effect [25]. Here, we address only numerical assessments of some essential factors affecting the impurity influx and focus on illustrating the behind physics/mechanisms associated with the common, essential geometric features of helical SOLs in non-axissymmetric devices rather than on simulating any specific discharges for a quantitative comparison with local diagnostics. Related experimental studies and experiment/modeling comparison results are referred to in Refs [22, 25, 26].

\subsection{Impurity source}

Both the ID in W7-AS and the HD in LHD have an open divertor structure and the first wall is made of stainless steel. Highenergetic CX-neutrals hitting the wall are a potential source of impurities by means of physical sputtering. A dense, cold island/stochastic SOL can move the CXneutrals to a lower energy band in the energy spectrum and thereby reduce the sputteringrelevant neutral flux. For a given input power entering the SOL, the SOL temperature drops with increasing SOL density, especially downstream of the maximum population of

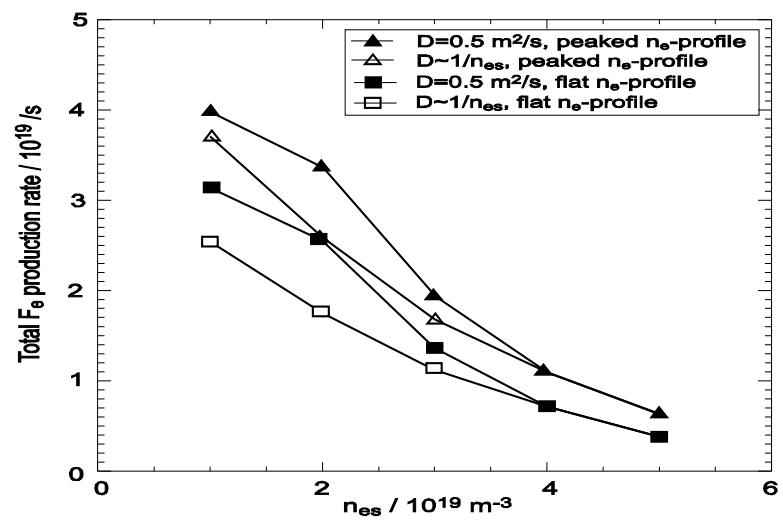

Fig.7 Sensitivities of high-energetic CX-neutrals to core profiles and cross-field transport coefficients as well as separatrix density, calculated by ECM3-EIRENE for W7-AS. recycling neutrals. Figures 7 and 8 show, respectively, the density dependence of the total calculated Fe yield sputtered by CX-neutrals for W7-AS [27] and LHD. Because of the existing uncertainties in wall conditioning, the Fe yield shown here should be regarded as a physics quantity for measuring the sputteringrelevant, high-energetic CX-neutral flux. For both W7-AS and LHD, the 3D code simulation results show that a dense, cold island/stochastic SOL can effectively reduce the high-energetic $\mathrm{CX}$-neutral flux and thereby the sputtered Fe yield. 


\subsection{Impurity SOL transport}

High-Z impurities like the wall-released $\mathrm{Fe}$ impurities can, even with a reduced source, cause significant radiation loss and contamination if they reach the confinement core region, preventing plasma from a high density operation. Impurity transport in the complex 3D SOLs of the W7-AS ID and the LHD HD has been studied using the EMC3/ EIRENE code based on a trace impurity model. The divertor-released carbon is used as a representative impurity species. There are reasons to do this. First, both the friction and ion thermal forces, which play governing roles in the parallel force balance, scale with $Z^{2}$. Thus, the induced parallel impurity flow velocities are largely independent of the charge and mass of the impurity species. Second, the multiple island structures in both machines distribute the background plasma flows over almost the whole SOL periphery in both the poloidal and toroidal directions. Thus, the whole SOL periphery lies downstream. Third, edge carbon spectroscopic measurements are available on LHD. Carbon neutrals are started from the targets with an initial energy of $0.1 \mathrm{eV}$, resulting in a penetration depth comparable with that of physically-sputtered Fe-impurities with a sputtered energy of several $\mathrm{eV}$. With a fixed carbon source strength, the plasma density is varied in order to check the carbon transport behavior under different SOL plasma conditions. The results for W7-AS are shown in figure 9 [11]. One sees a sharp drop of the carbon separatrix density with increasing the plasma density in the range from 1 to $4 \times 10^{13} \mathrm{~cm}^{-3}$, meaning that a dense SOL plasma has a retention effect on the carbon impurities. A similar effect has been also predicted for the stochastic layer in LHD [12]. Figure 10 shows how the carbon density profiles throughout the stochastic layer in the LHD HD change from peaked to hollow with increasing the plasma separatrix density. A parallel-force balance analysis based on the 3D simulations shows that the net force acting on impurities can, with increasing the SOL density, be changed from thermal-force dominated to friction dominated, leading to reversal of the convective impurity flow from

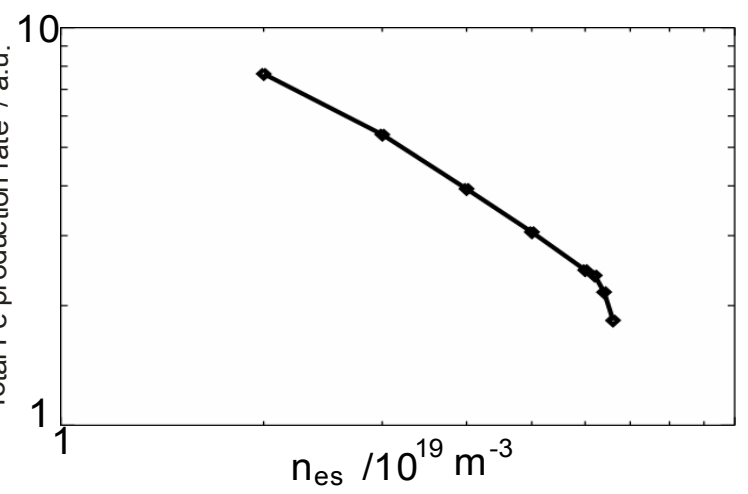

Fig.8 Calculated Fe-production rate as a function of $n_{\text {es }}$ in LHD.

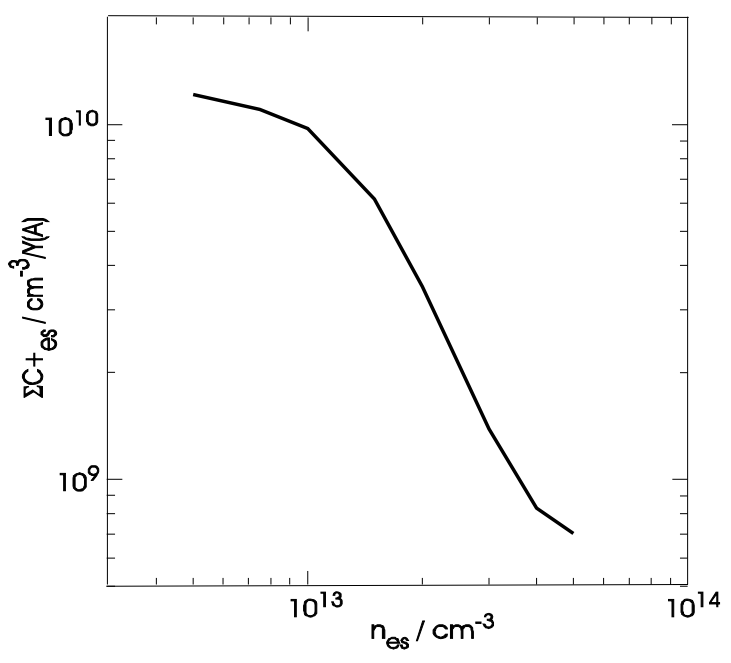

Fig. 9: Carbon separatrix density in W7$A S$ as a function of $n_{\text {es }}$ from $3 D$ simulations

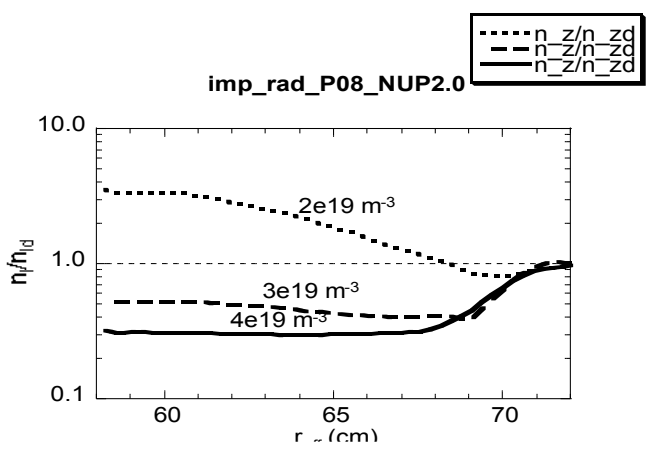

Fig.10: Radial profiles of total carbon density $n_{I}$ normalized by downstream density $n_{I d}$ in ergodic layer of LHD for different background plasma densities, obtained from 3D modelling. $r_{\text {eff }}$ is determined by cylindrical approximation of the volumes enclosed by each radial surface in the computational domain. 
inwards- to outwards-directed [9]. This is the reason for the reduced carbon density at the inner separatrix under high density conditions.

The island screening potential of intrinsic impurities is also examined for the standard ID of W7-X [20]. Using the calculated background plasmas shown in figures 5 and 6, test carbon impurities are sampled on the targets following the deposition distributions of the background ions calculated by the EMC3 code for individual cases. With a fixed total sputtered flux of 1 A $\left(1 / 1.6 \times 10^{-19}\right.$ particles/s), carbon atoms are started with mono-energies of $\mathrm{E}_{0}=0.1,1$ and $10 \mathrm{eV}$, respectively, covering both chemical and physical sputtering processes. Figure 11 shows the dependences of the averaged carbon density at the inner separatrix, $\mathrm{n}_{\mathrm{cs}}$, on $\mathrm{n}_{\mathrm{es}}$ and $\mathrm{E}_{0}$. In all the selected $\mathrm{E}_{0}$-cases, $\mathrm{n}_{\mathrm{cs}}$ drops sharply in the $\mathrm{n}_{\mathrm{es}}$-range from 1 to $2 \times 10^{19} \mathrm{~m}^{-3}$. Once friction becomes dominating, $\mathrm{n}_{\mathrm{cs}}$ reaches a minimum. The slight recovery of $\mathrm{n}_{\mathrm{cs}}$ in high $\mathrm{n}_{\mathrm{es}}$-range is due to the gradually-reduced streaming velocities of the back ground ions. Higher-energetic carbon neutrals penetrate more

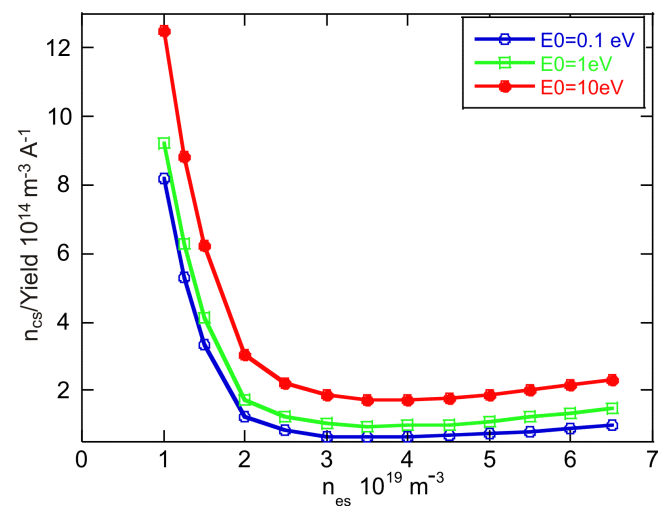

Fig. 11: Sensitivities of carbon density at the inner separatrix, $n_{c s}$, to $n_{e s}$ and initial energy of the sputtered carbon atoms, $E_{0}$, for W7-X $n_{c s}$ is normalized to a total carbon production rate of $1 A$. deeply into the SOL, causing an overall increase in $\mathrm{n}_{\mathrm{cs}}$. This means that the islands have a weaker screening efficiency on high-energy impurities than on low-energy ones. Nevertheless, the $\mathrm{n}_{\mathrm{es}}$-dependence is largely independent of $\mathrm{E}_{0}$. Higher SOL densities lower the energy of the projectiles onto solid surfaces and thereby the initial energy of the physicallysputtered impurities. Thus, $\mathrm{E}_{0}$ should decrease with raising $\mathrm{n}_{\mathrm{es}}$. Taking this effect into account would even steepen the $\mathrm{n}_{\mathrm{cs}}$ drops shown in figure 11. The strong drop in $\mathrm{n}_{\mathrm{cs}}$ is associated with a transition from thermal-force to friction-dominated impurity transport in the SOL, as understood from W7-AS and LHD, which will be analyzed bellow in some detail for W7-X. This transition is sharper in $\mathrm{W} 7-\mathrm{X}$ because of the high recycling regime.

A lower $\mathrm{n}_{\mathrm{es}}$-boundary is set at $1 \times 10^{19} \mathrm{~m}^{-3}$ in figure 11 to exclude the low SOL collisionality cases where the ratio between the connection length and the ion/electron mean free path length, $L_{c} / \lambda_{i, e}$ is less than 10 . As $n_{e s}$ is increased to $2 \times 10^{19} \mathrm{~m}^{-3}$, the ratio of $L_{c} / \lambda_{i, e}$ in the divertor region sharply to 100 . It has been checked that, in almost the whole island region, the $\lambda_{\mathrm{i}, \mathrm{e}} / \lambda_{\mathrm{Ti}, \mathrm{Te}}$ ratio $\left(\lambda_{\mathrm{Ti}, \mathrm{Te}}=\right.$ the parallel $\mathrm{T}_{\mathrm{e}}, \mathrm{T}_{\mathrm{i}}$ scale length) is well below unity within the parameter range investigated. Thus, kinetic corrections for the thermal force coefficients (see e.g. [28] and the references therein) seem not to be necessary. A proper assessment of the validity of the fluid approach in this collisionality range is beyond the scope of this paper. The following analysis, which will be based on fluid approximations, is focused on illustrating the physics behind the numerical findings shown in figure 11, leaving the absolute numbers there open for experimental confirmations or corrections by more precise physics models when available in future.

In a collisional SOL with small impurity concentrations, the following balance is believed to hold among the controlling parallel forces acting on impurities (see e.g. [28]):

$$
\frac{m_{I} n_{z}}{\tau_{Z i}}\left(V_{Z / /}-V_{i / l}\right)=-\nabla_{/ /} n_{Z} T_{Z}+n_{Z} Z e E_{/ /}+0.71 n_{Z} Z^{2} \nabla_{/ /} T_{e}+n_{Z} C_{i} \nabla_{/ /} T_{i}
$$

where the parallel electric field $E_{/ /}$is determined by electron momentum transport as

$$
n_{e} e \mathbf{E}_{/ /}+\nabla_{/ /} n_{e} T_{e}+0.71 n_{e} \nabla_{/ /} T_{e}=0 .
$$

Inserting eq (1) into eq (2), one obtains 


$$
V_{Z / /}=V_{i / /}+0.71 Z(Z-1) \frac{\tau_{Z i}}{m_{I}} \nabla_{/ /} T_{e}+\left(C_{i}-1\right) \frac{\tau_{Z i}}{m_{I}} \nabla_{/ /} T_{i}-\frac{\tau_{Z i} Z}{m_{I} n_{e}} \nabla_{/ /} n_{e} T_{e}-\frac{\tau_{Z i} T_{i}}{m_{I} n_{Z}} \nabla_{/ /} n_{Z}
$$

where $T_{z}$ has been replaced by $T_{i}$. The last term in eq (3) represents a diffusive process, tending always to smooth the density distribution along field lines. The other terms on the right side of eq (3) are convective contributions from the background plasma, which determine the form of the impurity density profile in the SOL. While $V_{\text {ill }}$ and $-V_{/ /} n_{e} T_{e}$ usually direct to targets, the temperature-gradient induced electron and ion thermal forces tend to draw impurities towards the confinement core periphery, where the power comes from. Thus, the balance between the two kinds of opposite forces determines the flow direction of the impurities and thereby the impurity density distribution in the SOL. All the terms are functions of the background plasma parameters, implying that the force balance can be influenced by changing the background plasma conditions. For each charge state, eq (3) describes the local force balance in a 3D space, thus being inconvenient for revealing the global impurity transport effects as shown by figure 11. For convenience, we define a quantity to measure the average parallel flow velocity of the impurities as

$$
\bar{V}_{Z / /}=\frac{\int n_{z} V_{Z / /} \cdot n_{e} V_{i / /} d v}{\int n_{z} n_{e}\left|V_{i / l}\right| d v}
$$

where the volume integrations should be performed over the whole SOL region. $V_{Z / /}$ is given by eq (3) omitting the last diffusive term. The dot-product between the impurity flux and the background ion flux has two meanings. First, the sign of the product gives the phase correlation in parallel velocity between the impurities and the background ions. Second, since the background ion flow dominantly results from the recycling process, averaging $V_{Z / /}$ weighted by $\boldsymbol{n}_{e} \boldsymbol{V}_{i / /}$ reflects the impurity mean flow velocity in the divertor region from the X-point to the targets, where the field lines leave the core periphery raidally way for targets. The average $V_{Z / /}$ as a function of $\mathrm{n}_{\mathrm{es}}$ for different charge states of carbon is shown in figure 12. The

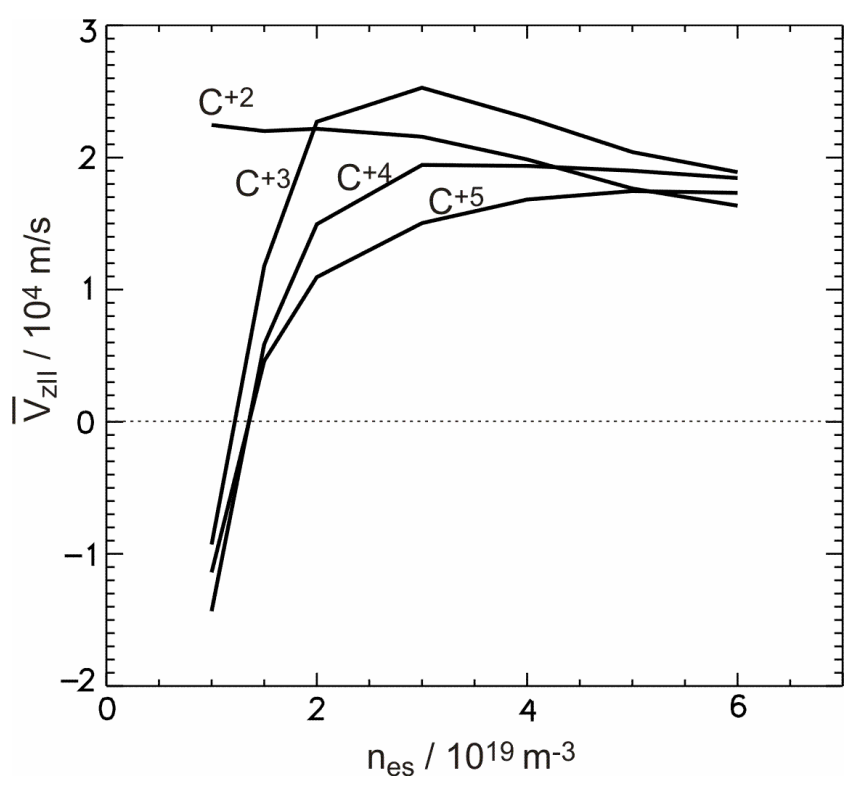

Fig. 12: $n_{e s}$-dependences of the average parallel flow velocities for difference carbon charge states in W7-X. downstream-residing $\mathrm{C}^{+2}$ ions show positive (target-directed) flow velocities throughout the whole $\mathrm{n}_{\mathrm{es}}$-range investigated, while the main contributors to the carbon density in the SOL, namely the $\mathrm{C}^{+3}, \mathrm{C}^{+4}$ and $\mathrm{C}^{+5}$ ions, exhibit sharp changes in the average parallel velocity from negative to positive within the $\mathrm{n}_{\mathrm{es}}$-range from 1 to $2 \times 10^{19} \mathrm{~m}^{-3}$. The $\mathrm{C}^{+6}$ and $\mathrm{C}^{+1}$ ions are not shown because of either their small population or their short lifetime. The flow reversal of the main carbon ion species accounts for the strong drop of the total carbon density at the inner separatrix as shown in figure 11.

It can be shown that the friction force and the ion thermal force play the governing roles in the force balance given by eq (1). In order for impurities to get a net flow velocity directed towards the targets, the friction force has to exceed the ion thermal force. This requires that $\left|\mathrm{M}_{\mathrm{i}}\right|>\lambda_{\mathrm{i}} / \lambda_{\mathrm{Ti}}$ [29], where $\mathrm{M}_{\mathrm{i}}$ is the Mach number of the background ions, or equivalently, that the ion parallel convective heat flux exceeds the conductive one [30], i.e. 


$$
\left|5 / 2 n_{e} V_{i / /} T_{i}\right|>\left|\boldsymbol{q}_{i}^{5 / 2} \nabla_{/ /} T_{i}\right|
$$

This condition can well be satisfied for the low charge state $\mathrm{C}^{+1}$ and $\mathrm{C}^{+2}$ ions of the target released carbon impurities, which, due to their short lifetime, reside near the targets where the background ions have high Mach numbers. This explains the weak $\mathrm{n}_{\mathrm{es}}$-dependence of the average flow velocity of the $\mathrm{C}^{+2}$ ions shown in figure 12. During the ionization processes to higher charge states, the carbon impurities can diffuse into regions of reduced Mach numbers where, depending on the SOL plasma condition, the ion thermal force can exceed the friction force drawing the impurities inwards. This is the case in a large part of the island SOL at low densities, high temperatures, accounting for the negative flow velocities of $\mathrm{C}^{+3}, \mathrm{C}^{+4}$ and $\mathrm{C}^{+5}$ at low $n_{\mathrm{es}}$-values shown in figure 12. Increasing $\mathrm{n}_{\mathrm{es}}$ and decreasing $\mathrm{T}_{\mathrm{i}}$ thereby will increase the ratio of cross-field to parallel heat conductive fluxes which scales with $n_{e} / T_{i}^{5 / 2}$ [9]. The former sets a bypass to the latter and can even, due to the small field-line pitches in helical SOLs, largely short-circuit the latter at high SOL collisionality conditions, leading to a strong reduction of the related thermal force [9]. This is reflected by the sharp changes in $V_{Z / /}$ from negative to positive for the $\mathrm{C}^{+3}, \mathrm{C}^{+4}$ and $\mathrm{C}^{+5}$ shown in figure 12 . Figure 13 shows how this happens in real space. Let us start from the left picture with $\mathrm{n}_{\mathrm{es}}=1.5 \times 10^{19} \mathrm{~m}^{-3}$. At this point, as shown in figure $11, \mathrm{n}_{\mathrm{cs}}$ has already been strongly reduced with respect to lower $\mathrm{n}_{\mathrm{es}}$ cases.

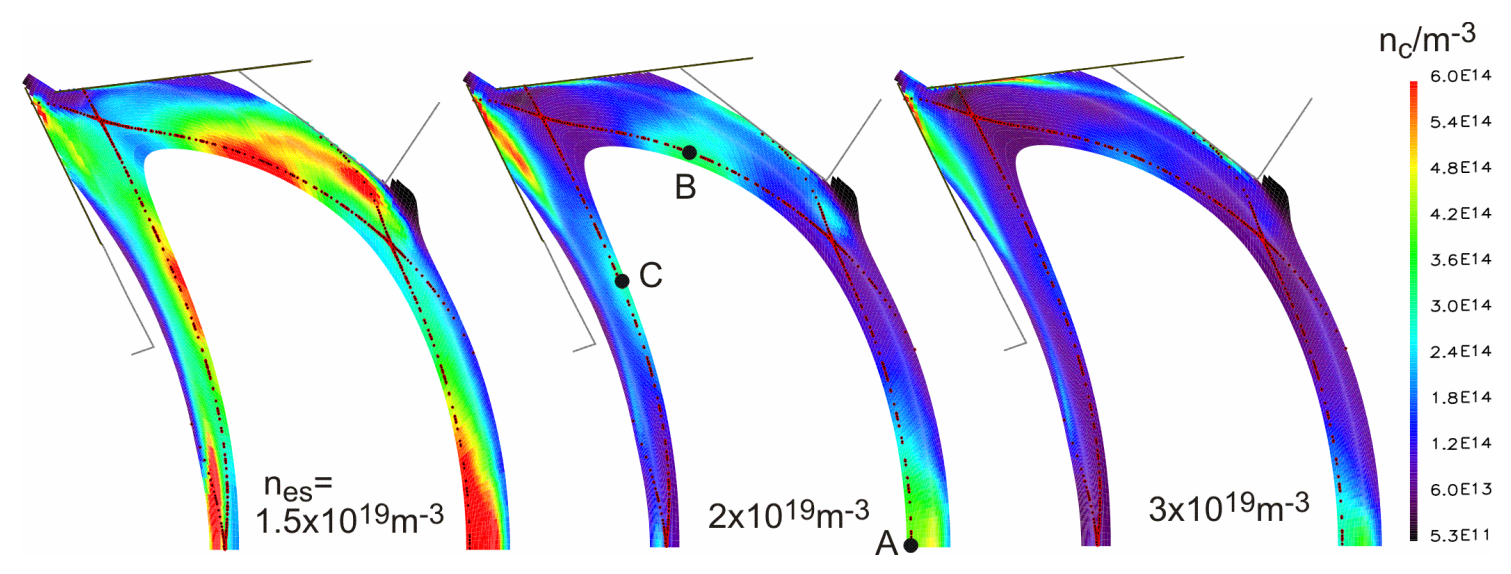

Fig. 13: Evolution of carbon density distribution with increasing the plasma density at the inner separatrix in W7-X. Carbon neutrals are started from the targets following the background ion flux deposition patterns. The total carbon source strength is 1 A. A, B and $C$ denote the upstream positions where the plasma stagnates.

A local force balance analysis shows that in this case the friction force is comparable with or even slightly exceeds the ion thermal force in the divertor region (see figure 12). As $n_{\mathrm{es}}$ increases to $2 \times 10^{19} \mathrm{~m}^{-3}$, the friction force becomes dominating over the whole divertor region from the X-point(s) to the targets, leading to a strong reduction of the carbon density there (see the middle picture in figure 13 in comparison with the left one). At the inner separatrix around the upstream positions indicated by A, B and C, the background ion flow is strongly reduced. In these regions, the ion thermal force, although already strongly reduced, always dominates, explaining why the carbon impurities accumulate there. With further increasing $\mathrm{n}_{\mathrm{es}}$, the carbon accumulation regions shrink (see the right picture), however, never disappear because of the existence of plasma stagnation regions.

\section{SOL conditions for high density operations in helical devices}

The density limit in helical devices is determined by impurity radiation [31, 32]. Core radiation results mainly from high-Z impurities released from plasma facing components. In the previous section we have shown how and under what conditions the divertors in the three helical devices can protect the confinement core region from contamination of intrinsic 
impurities. Using the divertors, both W7-AS and LHD have reached their respective record densities. Light impurities (mainly the target-released carbon) take the role of heavy impurities and the radiation origin shifts from core to edge, pre-limiting the plasma density due to Marfe-like radiation instabilities in detachment states.

Detachment is a SOL phenomenon, meaning that the Marfe-related density limit actually defines the maximum operational density for the SOL rather than for the core plasma. As in W7-AS and LHD the core density generally increases with $\mathrm{n}_{\mathrm{es}}$, the highest core densities were usually obtained just before onset of Marfes in both machines. While the $\mathrm{n}_{\mathrm{es}}$ limit can be well determined by Marfe formation, the core density achieved at the maximum $\mathrm{n}_{\text {es }}$ does not have the meaning of 'density limit' because there is no physics process which has been already set in and really limits the core density. Even in the internal diffusion barrier (IDB) regime in LHD [33], where a central density of $1 \times 10^{21} \mathrm{~m}^{-3}$ was obtained with massive central fuelling by pellet injection, no plasma-destroying, thermal or MHD instabilities originating in the core were observed. Thus, it has been expected that an even stronger pellet fuelling, which will be allowed after the improvement of the present pumping system by divertor closure in LHD, would increase the central density further. W7-AS has obtained its maximum density $\bar{n}_{e}=4 \times 10^{20} / \mathrm{m}^{3}$ in the high-density $\mathrm{H}$-mode $(\mathrm{HDH})$ [23]. As shown by EMC3/EIRENE simulations, the HDH plasmas were mainly fed by recycling neutrals. The core fuelling rate increases with $\mathrm{n}_{\mathrm{es}}$ in the detachment regime. Thus, the highest $\overline{\boldsymbol{n}}_{\boldsymbol{e}}$ was obtained in deeply detached plasmas just before onset of Marfe-like radiation instabilities, where the recycling neutrals have the highest core-fuelling rate [9]. Additional pellet fuelling in combination with particle pumping was not tried in W7-AS. High density plasmas in both devices are obtained under SOL plasma conditions with friction-dominated impurity transport predicted by the 3D code. Thus, operating the SOL plasma in the friction-dominated impurity transport regime, providing sufficient core particle fuelling and controlling the SOL density to avoid Marfe formation by efficient particle pumping are crucial conditions for achieving high density plasmas in helical devices, for which both the W7-AS ID and the LHD HD have demonstrated their great success. In the following, we give a numerical assessment for the W7-X ID in this regard.

In order to identify the detachment regime in W7-X, the calculations shown in figures 5, 6 and 11 have been repeated by adding the target-released carbon impurities. The sputtering coefficient is fixed to be $2 \%$, typically used in detachment simulations made for W7-AS. Figure 14 shows the total carbon line radiation as a function of $\mathrm{n}_{\mathrm{es}}$. A drastic change in carbon radiation occurs at $n_{\mathrm{es}} \sim 3 \times 10^{19} \mathrm{~m}^{-3}$, indicating the transition to detachment. This is also reflected by the sudden drop in $\Gamma_{\text {recy }}$ and $n_{\text {ed }}$ shown in figure 15 . Before detachment, the $\Gamma_{\text {recy }}$ and $n_{\text {ed }}$ curves do not differ very much from those shown in figures 5 and 6 without impurities, as the carbon radiation does not make significant contributions to the power balance in this $\mathrm{n}_{\mathrm{es}}$-range. Thus, the discussion of the high recycling regime and the impurity retention effect in the previous section is not affected by adding the impurities. Once the radiation exceeds $50 \%$ of the SOL power $(10 \mathrm{MW})$, the growing of $\Gamma_{\text {recy }}$ and $\mathrm{n}_{\text {ed }}$ is stopped and then they start to fall. The reached maximum $\mathrm{n}_{\mathrm{ed}}$ is by more than factor 3 higher than $\mathrm{n}_{\mathrm{es}}$. Note that $\mathrm{n}_{\mathrm{ed}}$ is always smaller than $\mathrm{n}_{\mathrm{es}}$ in W7-AS, even at rollover point. With the given, machine-size-scaled SOL powers for W7-AS and W7-X, the threshold upstream density for detachment transition is almost halved in W7-X with respect to W7-AS. Without justification for why the SOL power should linearly scale with the area of the LCFS for comparison, we make here a general conclusion i. e. that the existence of a prior high recycling regime in W7$\mathrm{X}$ allows earlier onset of detachment in the $\mathrm{n}_{\mathrm{es}}$-space. Without going in details of the detachment regime, which will be presented in a separate paper, figures 14 and 15 in combination with the impurity screen effects shown in figure 11 show that, for the given computational setup, there exists a narrow $\mathrm{n}_{\mathrm{es}}$-window from $2 \times 10^{19}$ to $3 \times 10^{19} \mathrm{~m}^{-3}$ within which 
the island divertor exhibits its optimum functionality in view of the high recycling flux and the good impurity shielding property.

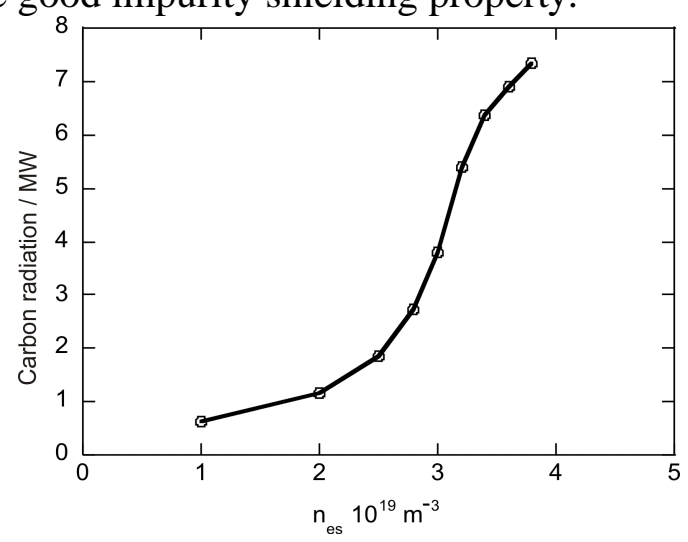

Fig. 14: Total carbon radiation as a function of $n_{e s}$ in $W 7-X$.

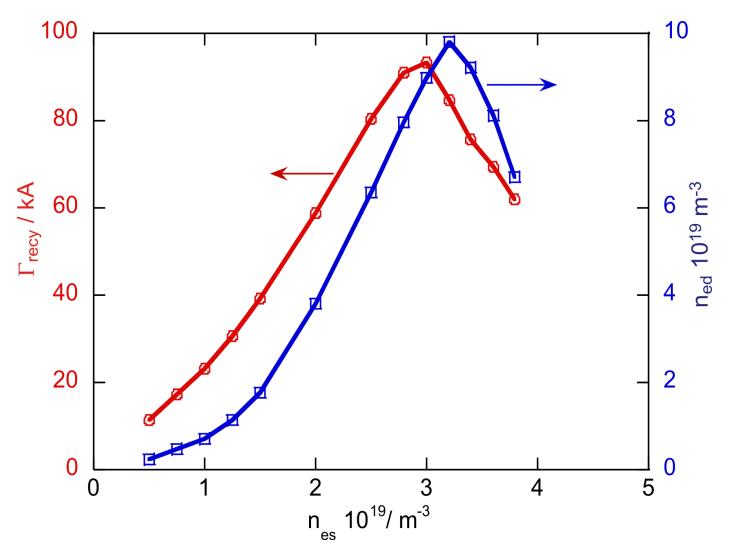

Fig. 15: Developments of $\Gamma_{r e c}$ and $n_{e d}$ with increasing $n_{e s}$ in $W 7-X$.

The recycling neutrals penetrating into the core attract our attention for two reasons. First, they provide a fuelling source for the core, usually exceeding that provided by NBI. Second, just through the source, they strengthen the SOL-core coupling. For the divertor plasmas in W7-AS, the core plasma was predominantly fed by the recycling neutrals and a close correlation in $\mathrm{n}_{\mathrm{es}}$-dependence between the measured $\bar{n}_{e}$ and the calculated core particle source was found. In comparison with W7-AS, the larger islands and the higher downstream densities in W7-X improve the divertor neutral screening efficiency, consequently reducing the core refuelling rates from the recycling neutrals. More precise calculations of the core refuelling rates would need exact knowledge of the core plasma profiles, which is beyond the scope of this paper. Instead, we define a simple core plasma by filling the region enclosed by a flux surface $\sim 2 \mathrm{~cm}$ inside the LCFS with hydrogen

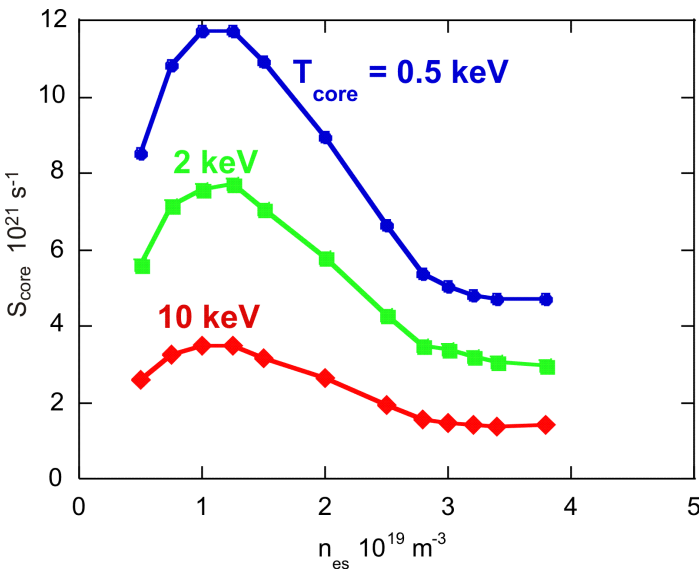

Fig. 16: Dependences of intrinsic particle core refuelling rates on core temperatures and $n_{e s}$ in $\mathrm{W} 7-\mathrm{X}$. plasma of constant temperature and density, with the temperature $\mathrm{T}_{\text {core }}\left(\mathrm{T}_{\text {core }} \equiv \mathrm{T}_{\mathrm{e}}=\mathrm{T}_{\mathrm{i}}\right)$ being varied to cover a reasonable range. We restrict our studies to dense core plasmas where the CX-neutrals have a mean free path shorter than the minor radius. Under this constraint, density is not a sensitive parameter for our results and is fixed to be $1 \times 10^{20} \mathrm{~m}^{-3}$. The total core refuelling rates $S_{\text {core }}$ as a function of $T_{\text {core }}$ and $n_{\mathrm{es}}$ are shown in figure 16. The three curves are different in level but similar in form. The overall decreases of $\mathrm{S}_{\text {core }}$ with increasing $\mathrm{T}_{\text {core }}$ within the given range result from the reduced ionization activities with respect to CX-processes in the core. On the other hand, the form of the curves is mainly determined by the influx of recycling neutrals into the core, thus reflecting the neutral screening efficiency of the edge islands and the recycling process. The maximum at $\mathrm{n}_{\mathrm{es}} \sim 1 \times 10^{19}$ is a consequence of competition between the increasing $\Gamma_{\text {rec }}$ and the gradually-improved neutral screening efficiency with raising $n_{\text {es }}$. The former effect dominates at lower $\mathrm{n}_{\mathrm{es}}$ while the latter at higher $\mathrm{n}_{\mathrm{es}}$. Similar behaviour is also predicted for W7-AS. Nevertheless, the drop of $S_{\text {core }}$ in W7-X is much stronger than that in W7-AS, showing improved neutral screening efficiency. W7-AS experiments have shown that the divertor plasmas which are controlled via gas-puffing using $\bar{n}_{e}$ as feedback cannot reach steady state 
in the $\mathrm{n}_{\mathrm{es}}$-region where $\mathrm{S}_{\text {core }}$ drops. This suggests that different plasma control strategies should be considered for W7-X in order to identify stable accesses to the above-mentioned narrow $\mathrm{n}_{\mathrm{es}}$-window for 'optimum' divertor performance. Furthermore, the improved divertor neutral screening and the expected higher core temperatures (more precisely, the temperatures in the neutral penetration region) in W7-X significantly reduce the recycling-neutral refuelling capability, implying the need for additional particle fuelling sources like pellets for achieving high density plasmas in $\mathrm{W} 7-\mathrm{X}$.

\section{Summary and discussion}

The island divertor tested at W7-AS and followed by W7-X and the helical divertor presently operated at LHD have a common geometric feature, i.e. that the low-order magnetic islands are the basic elements forming the divertor SOLs, providing a basis for a reasonable comparative divertor transport study. EMC3/EIRENE simulations in comparison with Thomson-scattering measurements have revealed the fundamental role of the low-order magnetic islands for plasma transport in the stochastic layer of LHD. Parallel plasma flows are modulated by the specific island structures in individual devices. Viscous-transport induced momentum loss due to proximity of opposite flows residing on adjacent island fans is considered to be the reason for the absence of a high recycling regime in W7-AS and LHD. The larger islands in W7-X avoid the viscous interaction between opposite flows. As a consequence, a high recycling regime is predicted to exist for $\mathrm{W} 7-\mathrm{X}$ from both $2 \mathrm{D}$ and $3 \mathrm{D}$ simulations. Regarding the divertor function on reducing the impurity influx, EMC3/EIRENE simulations show that a dense SOL plasma can significantly reduce the wall impurity release induced by the CX-neutrals escaping from the open divertors in both W7-AS and LHD. Moreover, the 3D code shows that, at high SOL collisionalities, the edge magnetic islands in all the three devices have a similar screening effect on intrinsic impurities. This happens when the cross-field heat conduction governs the ion energy transport across the islands under high density, low temperature conditions. In this case, the friction force dominates over the ion thermal force, dragging impurities outwards. These numerical results are supported by the strongly reduced core radiation at high densities observed in both W7-AS and LHD. With reduced impurity contents under divertor conditions, both devices have obtained their respective highest record densities which are limited by thermal instabilities induced by light impurity line radiation from the edge.

On the basis of the experience gained from the W7-AS and LHD divertor experiments and simulations, the SOL plasmas conditions for high density operations in W7-X is analysed, as a first step, for the standard divertor configuration. The appearance of a high recycling regime in W7-X shifts the detachment transition to lower SOL densities, significantly narrowing the operational SOL-density window of friction-governing impurity transport regime. The large islands and the strongly increased downstream densities in the high recycling regime improve the divertor neutral screening efficiency and, in spite of the enhanced recycling flux, reduce the core refuelling rates of the recycling neutrals to a level comparable to that predicted for the much smaller W7-AS device. This implies the need of external central fuelling pellets as already used in LHD in order to achieve high density plasmas in W7-X. In this case, the divertor pumping capability, which has been numerically studied for pure NBI sources [34], has to be increased accordingly in order to avoid an earlier onset of Marfe-like instabilities.

\section{References}


[1] R. König et al., PPCF 44, 2365 (2002)

[2] P. Grigull et al., PPCF 43, A175 (2001)

[3] H. Renner et al., Nucl. Fusion 40, 1083 (2000)

[4] N. Ohyabu et al., Nucl. Fusion 34, 387 (1994)

[5] Y. Feng et al., J. Nucl. Mat. 266-269, 812 (1999)

[6] D. Reiter, Fusion Sci. Technol. 47, 172-86 (2005)

[7] J. Miyazawa et al., Nucl. Fusion 46, 532 (2006)

[8] M. Kobayashi et al., J. Nucl. Mater. 363-365 294 (2007)

[9] Y. Feng et al., Nucl. Fusion 46, 807 (2006)

[10] Y. Feng et al., Nucl. Fusion 48, 024012 (2008)

[11] Y. Feng et al., 32 ${ }^{\text {nd }}$ EPS, vol 29C (ECA), P1.012 (2005)

[12] M. Kobayashi et al., Contrib. Plasma Phys. 48, 255 (2008)

[13] R. Brakel et al. in Fusion Energy 2002 (Proc. 19th Int. Conf. Lyon, 2002) (Vienna: IAEA) CD-ROM file EX/C5-4 and

http://www.iaea.org/programmes/ripc/physics/fec2002/html/fec2002.htm

[14] Y. Nakamura et al., Nucl. Fusion 43, 219 (2003)

[15] M. Kobayashi et al., "Model prediction of impurity retention in ergodic layer and comparison with edge carbon emission in LHD”, $18^{\text {th }}$ inter. Conference on Plasma-Surface Interactions in Controlled Fusion Devices, Toledo, Spain, 2008, O-3

[16] A. Komori et al., Nucl. Fusion 45, 837 (2005)

[17] T. Morisaki et al., J. Nucl. Mater. 337-339, 154 (2005)

[18] M. Kobayashi et al., Contrib. Plasma Phys. 46, 527 (2006)

[19] M. Kobayashi et al., Fusion Sci. Technol. 52, 566 (2007)

[20] Y. Feng et al., "EMC3/EIRENE Transport Modelling of the Island Divertor in W7-

X',35th EPS, Hersonissos, Crete, Greece, 2008, P2.085

[21] J. Kisslinger et al., EPS 19C, II, 149 (1995)

[22] R. Burhenn et al. Nucl. Fusion 49 No 6 (June 2009) 065005 (8pp)

[23] K. McCormick et al, Phys. Rev. Lett. 89 (2002) 015001

[24] R. Burhenn et al., Fusion Science and Technology, 46, 115(2004)

[25] M. B. Chowdhuri et al., Physics of Plasmas, 16, 062502(2009)

[26] M Kobayashi et al. in Fusion Energy 2008 (Proc. 22nd Int. Conf.

Geneva, 2008) (Vienna: IAEA) CD-ROM file EX/9-4 and

http://www-naweb.iaea.org/napc/physics/FEC/FEC2008/html/index.htm

[27] Y. Feng et al., J. Nucl. Mater., 363-365, 353 (2007)

[28] P. C. Stangeby, The Plasma Boundary of Magnetic Fusion Devices, Plasma Phys. Ser., Institute of Physics Publishing (2000)

[29] J. Neuhauser et al., Nucl. Fusion 24, 39 (1984)

[30] S. I. Krashenninikov, A. S. Kukushinkin and T. K. Soboleva, Nucl. Fusion 31, 1455 (1991)

[31] L. Giannone et al., Plasma Phys. Contr. Fusion 45(9) 1713-1731 (2003)

[32] S. Sudo et al., Nucl. Fusion 30, 11 (1990)

[33] H.Yamada et al, Plasma Phys. Control. Fusion 49 (2007) B487.

[34] D. Sharma et al. Nucl. Fusion 45, 825 (2005) 